\title{
Artelogie
}

Recherche sur les arts, le patrimoine et la littérature de l'Amérique latine

$8 \mid 2016$

Transgression dans les arts / transgression des arts

\section{Transgression dans les arts / transgression des arts. Introduction}

Gaspar Paz, Aline Miklos et Dominique Chateau

\section{OpenEdition}

\section{Journals}

Édition électronique

URL : http://journals.openedition.org/artelogie/419

DOI : 10.4000/artelogie.419

ISSN : 2115-6395

\section{Éditeur}

Association ESCAL

\section{Référence électronique}

Gaspar Paz, Aline Miklos et Dominique Chateau, «Transgression dans les arts / transgression des arts. Introduction », Artelogie [En ligne], 8| 2016, mis en ligne le 26 janvier 2016, consulté le 23 septembre 2020. URL : http://journals.openedition.org/artelogie/419; DOI : https://doi.org/10.4000/ artelogie.419

Ce document a été généré automatiquement le 23 septembre 2020.

Association ESCAL 


\title{
Transgression dans les arts / transgression des arts. Introduction
}

\author{
Gaspar Paz, Aline Miklos et Dominique Chateau
}

Nous remercions énormément l'ensemble de l'équipe d'Artelogie, surtout Edgar Vidal qui a été toujours très soucieux avec nous. Notre grand merci également à Cristina Moura, qui a traduit les textes de Dominique Château et Raoul Kirchmayr vers le portugais, et à tous les évaluateurs qui nous ont aidé à sélectionner les textes publiés ici. Sont-ils:

Adilson Florentino (UNIRIO), Aline Prúcoli (UFES), Amélia Siegel Corrêa (UFPR), André Queiroz (UFF), Camila Bechelany (EHESS), Camila Maroja (Brown University), Carine Lemouneau (Paris 1- Sorbonne), Carolina Amaral (USP), Christine Frerot (EHESS), David Ruiz Torres (UFES), Eduardo Dimitrov (UNB), Ignacio del Valle Dávila (UNICAMP) Lilian Santiago (UNIFESP), Mara Burkart (CONICET-UBA), Matías Allende Contador (Museo de Arte Contemporáneo de la Universidad de Chile), Mônica Zielinsky (UFRGS), Ricardo Fabbrini (USP), Rosângela de Jesus (UNILA), Tânia Navarro Swain (UNB), Tiago Barros (IFRJ), Tiago Machado de Jesus (USP), Vincenzo Cambria (UNIRIO).

1 Diverses formes de tensions (entre la norme et l'exception, la moralité et son contraire, le classique et le populaire, l'officiel et l'officieux) sont à l'œuvre dans l'histoire de l'art latino-américain, et la transgression y émerge comme une sorte de saturation des situations limites. La transgression met en scène l'opposition entre ces tensions, soit par un nouvel élan d'imagination et de créativité, soit par la disparition des événements. C'est ainsi que l'idée de transgression peut être considérée comme un moteur de transformation dans des situations diverses, compte tenu du fait que l'esprit critique renaît en un "principe de contestation $»^{1}$ (Maurice Blanchot, 2010), se définissant comme un effort de penser et d'agir d'une manière différente.

2 Pour ce qui concerne les arts, ces attitudes ont été vécues dans la critique de la normativité, la hiérarchie des valeurs et l'interdiction; en soulignant les limites du langage lui-même ${ }^{2}$. A partir de ces réflexions, nous avons constaté l'apparition d'œuvres qui impliquent au moins trois formes de transgressions : l'art qui dépasse les règles de l'art, l'art qui détruit les tabous, et l'art en tant que résistance politique. 
3 En Amérique latine, la rencontre entre les différentes cultures a généré, concernant l'art et les autres formes d'expression culturelle ou artistique, un processus de métissage et d'hybridation des formes. Ces événements, marqués par la violence et les circonstances contradictoires imposition/résistance et soumission/transgression, sont souvent le reflet d'affrontements entre colonisateurs et colonisés. En ce sens, ces relations sont caractérisées par l'acceptation, le rejet et l'incorporation de nouveaux éléments qui ont conduit à l'émergence de diverses formes de syncrétisme. De ce point de vue, la transgression peut être considérée comme le résultat de ces conflits, surtout quand elle est liée au processus de métissage et aux questions politiques ou morales c'est le cas dans les nombreuses controverses qui entourent la représentation de la Vierge avec des traits métissés, ou dans la toile du peintre académique brésilien Pedro Américo, «A Carioca ", très souvent objet de débats pour sa dimension érotique.

4 Au XIXe siècle, avec la création des premières Académies d'art en Amérique latine, les conflits entre art officiel et art populaire, entre tradition classique et styles de la modernité, ont surgi avec l'émergence de peintures historiques et de caricatures subversives.

5 Cette relation entre le Vieux et le Nouveau Monde ne s'est pas arrêtée avec l'indépendance des colonies, et elle subsiste jusqu'à la fin du XIXe siècle et le début du XXe siècle, avec l'émergence des avant-gardes locales. Ces mouvements ont été suscités par le désir de transvaloration des valeurs esthétiques et éthiques, par la rupture avec le classicisme et l'hégémonie de la politique colonialiste. Dans ce contexte, la transgression devient l'un des moteurs fondateurs des mouvements artistiques comme le muralisme mexicain, le modernisme brésilien ou le groupe cubain Minorista au cours des années 1920.

$6 \mathrm{Si}$, dans la première moitié du XXe siècle, la transgression a essentiellement signifié un désir de transformation, sa présence s'intensifiera plus tard dans les manifestations contemporaines dont les impératifs de singularité et d'authenticité incitent une grande partie des artistes à adopter la transgression comme posture indissociable de la création.

7 Dans de nombreux pays d'Amérique latine, les années 1960 et 1970 ont été marquées par l'émergence de l'art conceptuel, du néo-concrétisme et de l'art expérimental. A ces changements esthétiques se sont ajoutés des mouvements de contestation politique et morale permettant d'observer l'apparition d'œuvres qui, pour œuvrer sur la réalité même, ont tenté de défier les limites entre réalité et fiction, voire d'interroger la relation du corps aux normes morales; aboutissant à des expressions extrêmes telles que les œuvres de Batato Barea (Argentine), Mónica Mayer (Méxique) e Enrique Lihn (Chile). Les artistes se sont efforcés de subvertir les contraintes régissant non seulement les champs artistiques, mais aussi l'ensemble de la vie quotidienne: comportements sexuels, croyances religieuses, relations de genre, habitudes comportementales, relations contradictoires à la fois économiques, politiques et idéologiques.

8 Pour l'art contemporain, ces actes transgressifs ont soulevé au moins deux types de réaction en Amérique Latine: la censure et l'incorporation (l'acceptation). Dans le premier cas, la nécessité de réagir à l'exception produite, selon Nathalie Heinich, est le grand paradoxe contemporain : quand les artistes remettent en question les frontières des musées, le droit, la morale et l'autonomie, le milieu artistique se métamorphose en augmentant ses limites pour incorporer ce type d'expression. En raison de ce besoin 
d'acceptation (par les artistes) et d'incorporation (par les institutions), le sens de la transgression se transforme souvent en acte banalisé. La discussion autour de l'art transgressif finit par dominer; et sa relation avec les institutions permet l'adaptation aux médias, au marché de l'art, au capitalisme industriel, à la critique d'art, à sa production et sa diffusion.

9 Dans le second cas, on doit aussi considérer que ces interventions transgressives génèrent souvent des conflits, provoquent la censure, la violence et la criminalisation. Il n'est pas rare que des œuvres, exemplaires aujourd'hui, aient été censurées par les dictatures militaires dans de nombreux pays d'Amérique Latine pour des raisons politiques, morales ou religieuses. En temps de crise, de ségrégation, de montée des préjugés et de l'arrogance, de guerres de pouvoir, d'intolérance et de manipulation des médias, on peut observer que ce débat va au-delà des discussions sur la liberté d'expression ou sur l'autonomie pénale de la production symbolique, car il concerne des questions tant politiques que sociales, et des oppositions notables surviennent entre les différents acteurs concernés.

Toutes ces implications ont motivé l'organisation du Colloque international Transgression dans les arts / Transgression des arts, programmé à l'Université de São Paulo en 2013. Le projet a suscité une collaboration entre le Département de philosophie de cette université et l'Université Paris 1 Panthéon-Sorbonne (ACTE Laboratoire CNRS / Université Paris 1 - Groupe d'études esthétiques) pour ce qui relève de l'étude des problèmes esthétiques et éthiques de la contemporanéité. Coordonné par Gaspar Paz et Olgária Matos, le colloque, financé par la FAPESP (Brésil), a réuni des chercheurs de différentes institutions, parmi lesquels: Dominique Chateau, Jacinto Lageira, Lilian Santiago, Raoul Kirchmayr, Renato Janine Ribeiro, Ricardo Fabbrini et Samuel Araujo. Les interventions se sont révélées d'une telle acuité qu'elles nous ont incités à poursuivre le débat. La participation de la chercheuse Aline Miklos (École des Hautes Etudes en Sciences Sociales - EHESS), s'est notamment avérée décisive pour l'approfondissement de ces questions et l'élaboration de ce numéro de la revue Artelogie. Ainsi, en reprenant les échanges du colloque, le dossier s'ouvre sur un entretien avec la philosophe et écrivaine Olgária Matos (USP), dans lequel elle discute des principaux points soulevés lors de la rencontre, tels que les expériences créatives, l'humanisme occidental, l'autonomie des arts, le droit à la différence, l'industrie culturelle, ou encore les conceptions éphémères et l'arrogance du monde capitaliste.

11 Trois autres textes publiés à cette occasion apportent de nouvelles dimensions théoriques sur le thème en question. Le premier, signé par le professeur Ricardo Fabbrini et intitulé « Esthétique et transgression: de l'art radical à l'art radicant », expose la transformation de certaines tendances contradictoires du panorama esthétique contemporain et les paradoxes de l'art relationnel. À cet égard, l'auteur confronte les thèses de Nicolas Bourriaud, François Lyotard et Jacques Rancière. Ainsi, il interprète l'idée de transgression au cœur de la recherche et de la critique artistique.

Puis, dans l'essai intitulé «L'artiste aujourd'hui : entre le désenchantement postmoderne et les rebonds de l'engagement. À propos d'une exposition tunisienne ", -, le professeur Dominique Chateau a mené une réflexion sur l'impact d'une exposition présentée au Palais de La Marsa d'Abdellia, à Tunis, à l'occasion de la 10e édition du Printemps des Arts (2012). La censure de cette exposition, au prétexte que certaines œuvres heurtaient les principes de l'Islam, a provoqué une controverse. En évoquant cet épisode, et à l'appui d'une certaine relecture des théories de l'art, Chateau examine la place de la création et de la 
liberté dans l'expression artistique. L'auteur évalue ainsi l'impact culturel et les valeurs sociales, dans la mesure où il identifie l'artiste comme un type social persistant, susceptible de présenter des créations déstabilisantes.

Ce premier ensemble de textes s'achève sur l'article du professeur Raoul Kirchmayr intitulé «La transgression dans l'art: vers une esthétique de la spectralité (la Nymphe de Freud) ». Il y interroge le statut esthétique et épistémologique du spectre dans l'horizon actuel, en soulignant ses caractéristiques politiques, sociales et culturelles. Il analyse ainsi la relation de l'image au temps présent, et reprend l'idée de transgression de l'ordre métaphysique à partir de la célèbre lecture, développée par Freud, du roman Gradiva. Une Fantaisie pompéienne, de Wilhelm Jensen. Les textes de Dominique Chateau et Raoul Kirchmayr, publiés ici en français et en portugais, ont été traduits par Cristina Moura. Contributions dont elle a livré oralement les traductions successives au cours du colloque (USP).

Le deuxième ensemble est constitué d'essais traitant de la notion de transgression au travers d'œuvres interrogeant l'hétéronormativité et la dichotomie entre mâle et femelle. Ici, le corps n'est pas uniquement considéré comme outil mais également comme scène principale de toutes ces transformations. Existe-t-il mâle et femelle? L'auteure Araceli Sanchez tente de rompre avec ce binôme - parmi d'autres tels que "sacré / profane; pur / impur; transgression / esclavage" - via la théorie de la complexité d'Edgar Morin. Chez lui, la nécessité d'ordonner des phénomènes de la connaissance et de rejeter toute forme de trouble, d'ambiguïté ou d'incertitude caractéristiques propres au phénomène complexe -, pousse les individus à constituer leur univers à partir de dualismes et de catégories immuables, d'où l'émergence d'une certaine forme d'aveuglement (Morin, 2005: 3). À partir de cette idée, Sanchez développe une réflexion sur quelques œuvres d'artistes mexicaines féministes telles que Monica Mayer et Elizabeth Romero.

Le texte de Talita Trizoli, tente une rupture avec la dichotomie homme-femme à partir de la réflexion de Judith Butler sur les identités de genre en performance, selon laquelle le sexe est considéré comme une expérience et une pratique en constante métamorphose (Butler, 2003). En analysant le travail Sergio et Simone de Virginia de Medeiros, Talita Trizoli s'appuie également sur certains auteurs de la théorie Queer et sur l'idée de Beatriz Preciado (PRECIADO, 2014) d'user de l'ironie et de la parodie pour déstabiliser les systèmes de savoir, en relevant le caractère subversif et transgressif de l'œuvre par rapport à l'hétéronormativité. (Maria Laura Rosa, quant à elle, utilise aussi certains auteurs de la théorie du genre pour discuter des œuvres de trois artistes argentins Ilse Fusková, Liliana Maresca et Batato Barea - réalisées juste après la fin de la dictature militaire en Argentine. Ici, l'engagement politique de ces artistes implique que leurs productions artistiques et leurs corps deviennent des instruments de subversion par rapport à la règle hétéronormative.

Les quatre textes suivants traitent de l'art dans sa relation au politique. Avec «Le théâtre militant: subversions y résistances pendant la dictature civique militaire en Uruguay (1973-1985) », Luciana Scaraffuni soumet une analyse très fine des formes de résistance des pratiques théâtrales uruguayennes. Autant d'actions critiques envers la dictature au pouvoir, menées dans l'objectif d'échapper à la domination et aux coercitions qui pesaient sur le domaine culturel. Des troupes de théâtre indépendantes ont ainsi créé des espaces de dialogue, encourageant la prise de conscience et la mobilisation de manifestants en réaction à la violence d'État et à la répression. 

fleuri dans d'autres langages artistiques, tels la poésie et les arts visuels. En soulignant quelques-uns de ces aspects, mais cette fois-ci à l'aune de la réalité politique brésilienne des années 1962-1969, Marcelo Mari reprend les lignes essentielles de l'itinéraire du poète et critique Ferreira Gullar. L'auteur rend compte de la dimension politique et de l'engagement de l'époque pour comprendre le point de vue de Gullar sur la crise de l'avant-garde brésilienne et les motivations qui ont poussé l'artiste à une réorientation esthétique. Marcelo Mari relève que l'artiste a rompu avec les expérimentations concrétistes en produisant de la poésie et du théâtre militants afin d'éveiller la conscience politique de la classe ouvrière. Il agissait comme si les limites du langage et de la réalité provoquaient en lui-même un certain pouvoir de transgression. Ces considérations ont motivé l'intérêt de l'artiste pour un réalisme différent du réalisme socialiste, du réalisme du pop art américain et du nouveau réalisme français.

Toujours avec la scène brésilienne des années 1960 et 1970 pour horizon, la chercheuse Maria de Fátima Morethy Couto évalue la période à partir de la transgression de modèles et de censure, fruits des impasses de la situation politique et économique du pays. Pour cette raison, selon l'auteure, de nombreux artistes et intellectuels se sont engagés dans le débat politique avec l'intention de proposer des alternatives à ce paysage hostile, marqué par la dictature militaire. Couto aborde ces questions en tenant compte de tout un parcours historique de la politique brésilienne. Ainsi, elle se demande également sur comment ce contexte a influencé les artistes et les critiques de l'époque.

Débora Ferreira et Cleverson Luiz Salvaro abordent le jeu politique, artistique et philosophique d'une autre façon dans l'article intitulé «Les contrevenants du jeu: un dialogue entre Artur Barrio et Vilém Flusser ». Ils développent leur lecture de 4 jours, 4 nuits (1970), de A. Barrio, artiste luso-brésilien, en analysant cette œuvre à l'aune des concepts de poiesis, jeu et joueur, forgés par Flusser. Pour les auteurs de cette contribution, les théories de Flusser, ainsi comme les expériences transgressives de Barrio ' s'éclairent les unes par rapport aux autres, ce qui rend plus clair le caractère expérimental et innovant de leurs ?) œuvres.' La production chez Barrio est un exemple viscéral qui se lie aux concepts de Flusser, puis il incarne le jeu, la déterritorialisation, la critique au produit artistique, en ayant comme désir la transposition des espaces établis et des conventions sociales. Son actuation, qui subvertit les canons traditionnels, représente davantage une lecture importante de la réalité socioculturelle latino-américaine.

Dans son essai «Sel sans chair - pour une esthétique du ghetto », Angela Grando se promène au fil des «circuits idéologiques » de l'artiste Cildo Meireles, en insistant sur sa façon de traiter la réalité et l'utopie. Meireles a un geste artistique spécial pour faire face à la politisation de l'esthétique et l'esthétisation de la politique. Dans le cas de l'installation "Sel sans chair ", l'artiste brésilien dénonce l'expropriation et le mépris vis-à-vis des communautés indigènes. à partir d'une expérience qui n'a rien de conventionnel, où il représente (avec intensité, perplexité et action) la plasticité et la sonorité de circonstances défavorables.

21 La quatrième section, composée de trois textes, soulève un débat sur la relation entre transgression et règles de l'art. Il est intéressant d'observer que dans chacun de ces textes la transgression se produit par le biais des «mouvements" et de «l'hybridation ». Lina X. Aguirre ouvre cet ensemble avec l'analyse des œuvres de deux artistes contemporains chiliens, affirmant que la rupture des paradigmes de l'art peut 
également se produire à travers des opérations de transformation ou de transit entre les médias artistiques, désignées sous le concept de transference. Pour l'auteur, l'utilisation récurrente d'outils numériques et de la technologie a permis de modifier plusieurs fois l'œuvre d'art, au point où les moyens artistiques se mélangent et se chevauchent, provoquant ce que la critique d'art Rosalind Krauss appelle la condition post-médium (KRAUSS, 2000).

Giuseppe Gatti Riccardi et Rafael Arce analysent deux romans où la transgression opère également dans l'hybridation des formes. Giuseppe Riccardi examine le roman Pour Eva (écrit dans les années 1980 et publié à titre posthume), d'Enrique Lihn. Riccardi suggère ici un dialogue entre littérature et arts plastiques; cet écrivain ayant révélé que certaines de ses fictions ont été inspirées par ses propres peintures. Pour Riccardi, la nature subversive de l'œuvre de Lihn réside dans la «carnavalisation » de la rupture avec les canons de l'orthodoxie esthétique et sexuelle, ainsi que dans le travestisme, artifice utilisé par l'auteur comme stratégie de création. Rafael Arce affirme lui aussi que dans Zama (1956), d'Antonio Di Benedetto, la transgression s'inscrit dans la forme ; cette œuvre pouvant être interprétée comme un déni du roman historique puisqu'elle viole tout le processus de vraisemblance dans la construction narrative. Attentif à l'humour e de ce roman, Rafael Arce conclut que le grotesque est suscité par un mouvement constant, passant de l'homme à l'animal, de l'animal au végétal, etc.

Le dernier groupe de communications porte sur l'institution et la critique d'art. Almerinda Lopez da Silva a ainsi analysé la réception critique de la production de Georges Mathieu au Brésil. L'auteur, qui a travaillé de façon sui generis le langage pictural et l'action performative, a suscité la controverse en rompant avec certains préceptes, lors d'une exposition présentée au Musée de Rio de Janeiro, en 1959. Ce fut une performance, unissant une grande toile abstraite, de la musique et de la danse afrobrésilienne. Motivées par ces actions, les critiques de l'époque ont évalué l'œuvre en la mettant en relation directe avec l'histoire et la production artistique au Brésil.

L'ensemble des textes publiés dans ce dossier révèle que les gestes artistiques et transgressifs ont pris différentes formes et significations au fil du temps, mais mais demeurent cependant des gestes confrontés à l'idée de limite. Il est également intéressant d'observer que ces gestes ont signifié bien davantage qu'une simple rupture de limites préétablies. De nombreux cas décrits ici ont abouti à la flexibilité, comme au déplacement des règles ou conventions. Donc, si dans un premier temps et à des fins didactiques, nous avons réparti les arts transgressifs selon trois modèles d'expression l'art porté au-delà des règles de l'art, l'art destructeur de tabous, l'art comme mode de résistance politique -, il apparaît évident que tous les types de transgression sont constamment mêlés. Ils ont en commun un principe de contestation et une nécessité impérieuse de changement.

On remarquera que ces différentes approches impliquent d'une manière ou d'une autre à des affrontements concrets de l'acte de transgression. Certaines de ses caractéristiques sont indélébiles pour l'héritage culturel considérable qu'elles ont suscité. Comment ne pas penser à Sade (mais aussi bien aux surréalistes et à Artaud) en démontrant «inlassablement la sottise, l'hypocrisie et la nullité de la vertu». (BLANCHOT, 2010: 83) ? Et si nous changions d'optique pour poursuivre le travail critique et théorique? Sigmund Freud a ouvert le chemin pour penser la transgression dans le domaine de la sexualité et de l'inconscient. Dans son sillage, Georges Bataille a articulé la gamme complète des spécificités énoncées par Freud. Ainsi, avec l'Érotisme et 
Documents, tout un domaine d'expertise s'est dévoilé aux critiques - historiens de l'art, anthropologues, archéologues, ethnologues, psychanalystes, entre autres. Dès lors, de nombreux intellectuels ont creusé le terrain défriché par Bataille - Michel Foucault, Maurice Blanchot et Georges Didi-Huberman, notamment.

Les nouveaux positionnements transcendent les impostures des institutions, la violence policière, la vacuité des relations sociales. En une époque, comme dirait José Américo Motta Pessanha, «marquée par l'accent mis sur les notions de rupture, de différence et de pluralité » (PESSANHA 1987 : 61). D'après Pessanha, les traits de la contemporanéité permettent d'apercevoir tout court les catégories de "multiplicité, de rupture, d'altérité » (PESSANHA 1987 : 61). Avec l'exposition de cette altérité, c'est tout un pan du despotisme social qui se retrouve affaibli, voire entamé. La régulation des pouvoirs et des droits sociaux établis la plupart du temps imposée légalement, instaure, comme l'a souligné Marilena Chauí, «la préservation des privilèges et de l'exercice de la répression ». (Chauí $2001: 14$ ). Selon elle « c'est pourquoi les lois semblent inopérantes, inutiles et incompréhensibles, faites pour être violées et non pour être transformées » (CHAUI $2001: 14$ ).

En évoquant quelques-unes des tendances liées à l'acte de transgression, nous souhaitons rendre justice aux inflexions d'un panorama sans précédant. Les implications de ce scénario sont vastes et configurent une nouvelle conscience historique visant à une compréhension critique des hypothèses esthétiques et de leurs implications sociales. Ces réflexions ouvrent de nouvelles façons de penser les arts de l'Amérique latine. De surcroît, elles constituent un corpus audacieux de propositions pour affronter les conflits et paradoxes des expressions de notre temps.

\section{BIBLIOGRAPHIE}

BATAILLE Georges, L'Erotisme, Paris, Les Editions de Minuit, 2001.

BATAILLE Georges, La littérature et le mal, Paris, Gallimard, 1957.

BLANCHOT Maurice, La condition critique, Articles 1945-1998, Paris, Gallimard, 2010.

BLANCHOT Maurice, Une voix venue d'ailleurs, Paris, Gallimard, 2002.

BUTLER Judith, Problemas de Gênero. Feminismo e Subversão da Identidade, Rio de Janeiro, Civilização Brasileira, 2003.

CHAUí Marilena, Escritos sobre a universidade, São Paulo, Editora UNESP, 2001.

DIDI-HUBERMAN Georges, “Como transgredir a forma ?”, in A semelhança informe : ou o gaio saber visual Segundo Georges Bataille. Tradução Caio Meira, Fernando Scheib e Marcelo Jacques de Moraes, 2015.

FOUCAULT Michel, "Prefácio à transgressão", in Estética : literatura e pintura, música e cinema. Organização e seleção de textos Manoel BARRos DA MOTTA. Tradução : Autran DOURADO, Rio de Janeiro, Forense Universitária, 2001. 
HEINICH Nathalie, Le triple jeu de l'art contemporain, Paris, Les Éditions de Minuit, 2006.

KRAUSS R.E., BROODTHAERS M., A Voyage on the North Sea: Art in the Age of the Post-medium Condition, New York, Thames \& Hudson, 2000.

MORIN Edgar, Introducción al pensamiento complejo, Barcelona, Gedisa, 2005.

NOVAES Adauto (org.), Libertinos e libertários, São Paulo, Companhia das letras, 1996.

PESSANHA José Américo Motta, "Cultura como ruptura”, in Tradição e contradição, Rio de Janeiro, Jorge Zahar editora/ Funarte, 1987.

PRECIADO Beatriz Paul, Manifesto Contrasexual. Práticas subversivas de identidade sexual, São Paulo/ Helsinki, N-1 edições, 2014.

STALYBRASS Peter e WHITE Allon, The politic and poetics of transgression, Ithaca, Cornell University Press, 1986.

\section{NOTES}

1. Michel Foucault fait référence à ce principe de contestation, en Blanchot, pour souligner une espèce de "probation des limites" (FOUCAULT, 2001 : 34). Pour ce sujet, voir également les essais suivants de Maurice Blanchot: "La condition critique", "Le discours philosophique" et "Quelques remarques sur Sade" (Blanchot, 2010).

2. Michel Foucault, dans le "Préface à la transgression" (FOUCAULT, 2001 : 28), à fin de discuter sur l'idée de sexualité, il met l'accent sur ces trois points suivants : la limite de la conscience, la limite de la loi et la limite du langage.

\section{RÉSUMÉS}

Diverses formes de tensions (entre la norme et l'exception, la moralité et son contraire, le classique et le populaire, l'officiel et l'officieux) sont à l'œuvre dans l'histoire de l'art latinoaméricain, et la transgression y émerge comme une sorte de saturation des situations limites. La transgression met en scène l'opposition entre ces tensions, soit par un nouvel élan d'imagination et de créativité, soit par la disparition des événements. C'est ainsi que l'idée de transgression peut être considérée comme un moteur de transformation dans des situations diverses, compte tenu du fait que l'esprit critique renaît en un "principe de contestation » (Maurice Blanchot, 2010), se définissant comme un effort de penser et d'agir d'une manière différente.

\section{AUTEURS}

GASPAR PAZ

UFES 
ALINE MIKLOS

CRAL-EHESS

Olgária Matos

USP

DOMINIQUE CHATEAU

Sorbonne 